\title{
PULSED WATER JET GENERATED BY PULSE MULTIPLICATION
}

\author{
Richard Dvorsky, Libor Sitek, Tomáš Sochor
}

Original scientific paper

First theoretical papers, summarizing the high mechanical energy cumulation when high-speed drops impact on the solid surface as a result of the water hammer effect, have been known since 1960's. Heymann has demonstrated that pressure maximum during the impact of a spherical drop of a liquid is several times higher than the presupposed maximum for the classical waterhammer effect. The mentioned maximum pressure exceeds the stagnation pressure of the continuous jet several times over. When developing devices for pulsed jet generation, a new generalization of the classical water hammer theory for high pressures has been introduced. Based on this a new patented principle of "pulse multiplication" has been formulated. The pulse multiplier is the source of high-pressure pulses with $100 \%$ depth of modulation of discharge velocity of a liquid jet. It enables to significantly increase the jet disintegration effect without addition of abrasives.

Keywords: high-pressure pulses; pulse intensifier; pulsed water jet; water hammer effect

\section{Pulsirajući vodeni mlaz dobiven multiplikacijom impulsa}

Izvorni znanstveni članak Prvi teorijski radovi, koji su opisivali stvaranje velike količine mehaničke energije kad kapljice velike brzine udare u čvrstu površinu kao rezultat hidrauličkog udara, poznati su još od 1960-tih. Heymann je pokazao da je maksimalni tlak kod udara loptaste kapljice tekučine nekoliko puta veći od pretpostavljenog maksimalnog u slučaju klasičnog hidrauličkog udara. Spomenuti maksimalni tlak nekoliko je puta veći od tlaka kontinuiranog mlaza u stanju mirovanja. Kod stvaranja uređaja za generiranje pulsirajućeg mlaza, za visoke je tlakove uvedena nova generalizacija klasične teorije hidrauličkog udara. Na tom je temelju zasnovan novi patentirani princip "multiplikacije impulsa". Multiplikator impulsa je izvor visokotlačnih impulsa sa $100 \%$ dubinom modulacije izlazne brzine tekućeg mlaza. Omogućuje značajno povećanje dezintegracijskog učinka mlaza bez dodavanja abraziva.

Ključne riječi: pojačavač impulsa; pulsirajući vodeni mlaz; rezultat hidrauličkog udara; visokotlačni impulsi

\section{Introduction}

The technology of high-energy liquid jet (Water Jet) $[1,2]$ (hereinafter "liquid jet") originated in 1971 in NASA laboratories [3, 4] for the purpose of cutting and machining of hard high-temperature ceramics without significant thermal influence on cutting surfaces. Thanks to this feature, the liquid jet is a tool with high application potential that has not been fully explored yet. New findings and applications are expected especially in the field of micronozzles with very narrow apeture, vapor microcavitation $[5 \div 8]$ and in the area of pulsed mode $[9 \div 13]$ where the water hammer effect multiplies its erosive efficiency and rapid changes of a velocity field result in an intense cavitation.

Since several recent decades, a very old idea of erosive water stream (coming from ore and mineral mining) has undergone a turbulent development. It has resulted in creation of highly sophisticated devices using the high-energy liquid jet as a relatively very precise machining tool [1]. The liquid jet enables processing of a wide range of materials, from minerals, metals and plastics to soft biological tissues. The current high level of the technology was achieved by design and manufacturing of high-pressure pumps with output pressure of thousands of MPa.

The high-energy liquid jet represents an extremely narrow free stream of a liquid flowing out of a sapphire or diamond nozzle. Its standard medium cutting speeds vary from values of approx. $600 \mathrm{~m} / \mathrm{s}$ up to the commercial maximum of approx. $1000 \mathrm{~m} / \mathrm{s}$, used in devices in the Flow Internantional Company. The current development of jets operating in the pulsed mode in the field of nanogrinding is enhanced by extreme disintegration efficiency of this type of jets caused by pressure multiplication through the water hammer effect and high intensity of cavitation in the liquid dispersion of a material being ground. In the pulsed mode, a significant cavitation field occurs in the liquid environment in the time-periodic areas of the pressure release.

Another specific feature of liquid jets is the time behaviour of the flow of the liquid through the nozzle. If the liquid flow in the nozzle varies periodically in time, the jet is generally modulated. The jets can be classified according to the depth of modulation as modulated jets, with the depth of modulation significantly smaller than $100 \%$ of the pump pressure, and pulsed jets, with very significant depth of modulation up to $100 \%$ of the total pressure value. It should be noted here that practically all current operating systems belong (from the point of view of primary actuating pressure) rather to medium pressures with medium discharge velocity. Fully functional pulsed jets of high pressures of hundreds of MPa have not yet been developed for practical technical applications.

\section{Comparison of effects of pulsed and continuous jets}

Erosive effect of the pure water jet in the continuous mode has proven to be not efficient enough for cutting of the majority of hard materials. Therefore, its local erosive effects are often multiplied by inserting of soft abrasive material into the stream of liquid flowing out [1]. There is a quite promising option to replace the erosive effect of solid abrasive material by implosion of cavitation bubbles at the interface between the liquid and the solid material.This is characterized by a very high volumetric density of a released mechanical energy [1]. In case of the currently used cavitation jets, there is a lack of both sufficient volumetric density of cavitation bubbles and kinetic energy of the water stream in cavitation mode The 
third prospective option how to increase the erosive effect of the water jet is the pulsed mode of the outflow of the pure liquid jet in a form of relatively compact macroscopic drops with dimensions comparable to the diameter of the outflow nozzle within the range of several tenths of millimeters.

First theoretical papers by Heymann [14] and Huang [15] have been known since late 1960's that summarize the high cumulation of mechanical energy during the impact of very fast drops on the solid surface as a result of the water hammer effect. Heymann has demonstrated that the pressure maximum during the impact of a spherical drop of a liquid is several times higher than the presupposed maximum given by the classical Zhukovskij equation for hydraulic shock. The mentioned maximum pressure already exceeds the stagnation pressure of the continuous jet several times over. The theoretical predictions were confirmed by experiments performed by Smith and Kinslow [16]. In addition to high maximum values of pressure, the erosive effect of the pulsed jet has been also improved due to extreme dynamics of periodic shock waves in the area of impact. It leads to high deformation speeds and increased probability of brittle fracture. One of other erosive mechanisms to be considered is the significant cavitation intensity when the jets flow out into a liquid.

\section{Experience with generating pulsed liquid jet gathered so far}

Basic classification of pulsed jets shows two completely different directions of the current development. The first group, called "pulsed guns", is formed by a series of successive individual pulses drops. This direction has its origin in machines able to generate individual pulses either by an explosion of explosives behind the actuating piston or by a very fast opening/closing of valves at the reservoir (blowdown water canon) of high-pressure liquid (approx. $400 \mathrm{MPa}$ ) [17]. The second group of devices is based on the modulation of discharge velocity of the originally continuous jet. In case of $100 \%$ depth of modulation, that is very similar to the mode of the "pulsed gun", various types of mechanical interrupters located behind the nozzle are usually used [18].Those mechanisms have not proven to be prospective enough for the future development due to high cavitation damage, energy loss and other complications.

From the point of view of practical application, methods of the internal modulation of discharge velocity, followed by a forced jet breakup behind the nozzle, seem to be much more important. The classical breakup driven by the Rayleigh mechanism [19] is caused by the amplification of shape defects due to surface tension effect. Unlike the classical breakup, the forced breakup that is influenced by the discharge velocity modulation based on the Pimbley's theoretical analysis [20] is dominantly caused by the periodical inertial dynamics of the liquid jet. While in the case of passive modulation, where especially acoustic resonance is employed [21], [22], relatively small depth of modulation is achieved and technically significant increase in erosive effects has not been proven yet, significant progress has been achieved using methods of an active flow modulation around the nozzle.

In 1990's the first equipment applicable in practice based on the high-frequency mechanical modulation of flow cross-section at the nozzle inlet was developed by Vijay [9]. However, its greater expansion has not been possible so far because of the necessity of modification of the nozzle that cannot be ingrated into already existing hydraulic systems using the classical nozzle. Secondly, only relatively low operating pressures in the order of tens of $\mathrm{MPa}$ can be used due to an extreme increase in technical problems with the sealing of the oscillating tip. An ultrasonic device, integrated into a standard inlet of pressured liquid and allowing to achive the pulsed mode at medium pressures, was developed by Foldyna and Švehla [23] in 2008.

\section{Classical theory of hydraulic shock and Zhukovsky formula}

The classical theory of hydraulic shock is based on the situation at the time of its origin. It was applied only to low-pressure processes in water supply networks. In the conditions of low pressures, the relative change of water density depending on the increase in pressure is negligible. Both the density $\rho$ and the corresponding velocity of the pressure wave $a$ can be considered to be constant

$$
\frac{\partial v_{x}}{\partial r}=0, \quad \frac{\partial v_{x}}{\partial \varphi}=0
$$

Based on the theoretical assumption of the total transformation of the kinetic energy of a water column of the length $L$ into the pressure energy, the following formula determining the pressure maximum $p$ of a shock wave was derived by N. E. Zhukovsky [24]

$p=\rho v a$.

In the case of validity of the Eq. (2), the liquid flowing through the tube of the length $L$ at the crosssectional velocity $v$ must be completely stopped for the period $\tau$ at the closure. The time period must be shorter than the time of return of the pressure wave $a$ reflected from the beginning of the tube

$a \tau \leq 2 L$.

And thus the condition of the total hydraulic shock with complete transformation of the kinetic energy into the pressure energy is formulated. The pressure wave flowing from the closure in the opposite direction to the flow towards the beginning of the tube is reflected from there and flows back towards the completely closed end.

The graph in Fig. 1 illustrates the conditions for the practical validity of the classical theory that correspond to real velocities of the flow in a water supply network. There is only one practical application of the hydraulic shock in history - i.e. in automatic pumps called water ram pumps. In the majority of cases, this phenomenon was rather undesired, as it could even result in cracking of the water supply tubes. Therefore, its eliminatation was 
necessary. This paper presents a new technical solution allowing the application of the hydraulic shock in the range of medium and high pressures.

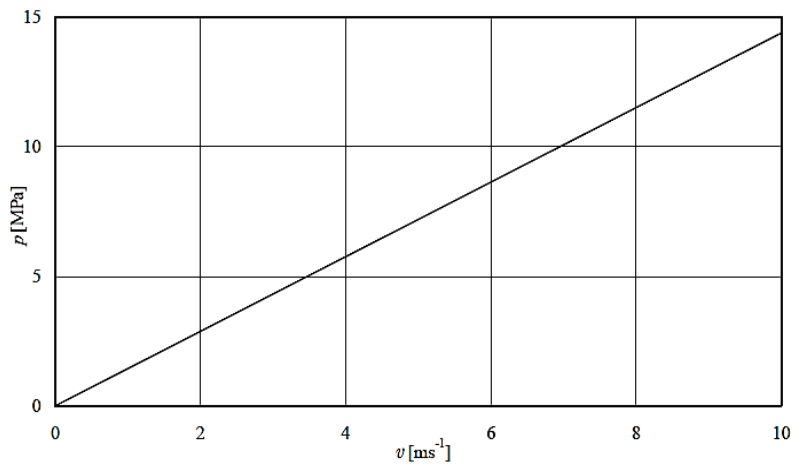

Figure 1 The graph of the relation between the amplitude of the pressure $p$ developed by the total hydraulic shock, based on the classical Zhukovsky equation, and the initial velocity of the liquid in the tube

\section{Generalization of hydraulic shock theory at high pressures}

The movement of compressible viscous liquid in a thin long shock tube of a circular cross-section can be described in cylindrical coordinates due to the rotary symmetry, see Fig. 2.

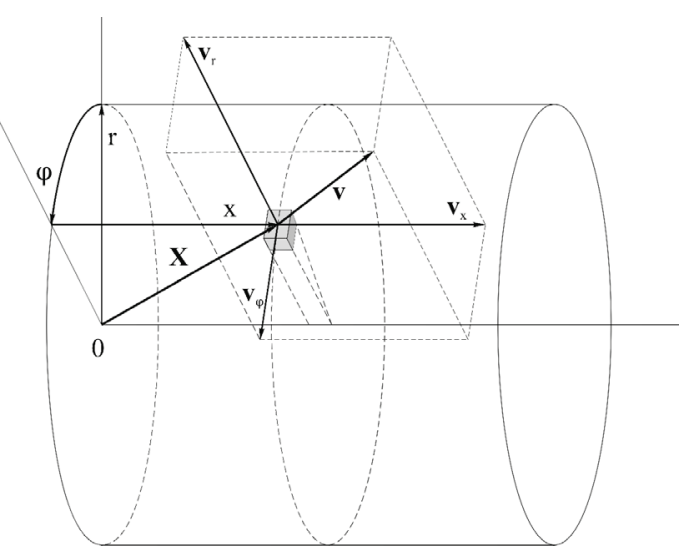

Figure 2 Scheme of the introduction of cylindrical coordinates of $\boldsymbol{X}(\boldsymbol{r}, \boldsymbol{\varphi}, \boldsymbol{x})$ in ashock tube and depiction of the vector $\boldsymbol{v}=\boldsymbol{v}_{\boldsymbol{r}}+\boldsymbol{v}_{\boldsymbol{\varphi}}+\boldsymbol{v}_{\mathrm{X}}$ of the local velocity of the liquid

The behaviour of state parameters of the liquid column (i.e. the pressure $p$ and the macroscopic velocity $v$ without turbulent fluctuations) is simplified by acceptance of the following model conditions:

I. Particles of the liquid move in the differential layer $\Delta x$ only in the axial direction:

$v\left(v_{x} \neq 0, v_{r}=0, v_{\varphi}=0\right)=v_{x}$.

II. For the given value of the axial coordinate $x$, the liquid has a constant velocity profile along the whole cross-section of the tube:

$$
\frac{\partial v_{x}}{\partial r}=0, \quad \frac{\partial v_{x}}{\partial \varphi}=0
$$

III. The velocity of the liquid flow through the tube of the radius $R=0,5 \mathrm{~mm}$ along the $x$ axis is uniquely representedby the mean "cross-sectional" velocity in the given point of the cross-section

$v \stackrel{\text { def }}{=} \frac{1}{\pi R^{2}} \int_{0}^{R} \int_{0}^{2 \pi} v_{x} \mathrm{~d} \varphi \mathrm{d} r=v_{x}$

This velocity reaches the maximum value of $200 \mathrm{~m} / \mathrm{s}$ at the beginning of the tube $x=0$ at the time $t=0$ and corresponds to the local acoustic velocity of the shock wave.

IV. The liquid density is homogeneous at any level of $x$ :

$\frac{\partial \rho}{\partial r}=0, \quad \frac{\partial \rho}{\partial \varphi}=0$

V. The studied barotropic liquid is the water with the pressure dependence of the density (see Fig. 3) given by the regression of compressibility data [25]

$$
\rho=\rho_{0} \mathrm{e}^{\gamma_{0} p-\beta p^{\frac{5}{3}}} \quad \begin{aligned}
& \rho_{0}=998,2 \mathrm{~kg} / \mathrm{m}^{3}, \\
& \gamma_{0}=4,83 \times 10^{-10} \mathrm{~Pa}^{-1}, \\
& \beta=2,94 \times 10^{-16} \mathrm{~Pa}^{-3 / 5}
\end{aligned}
$$

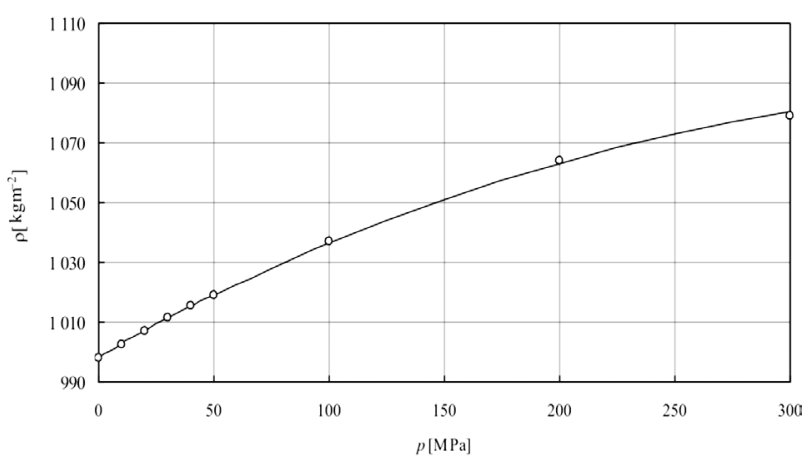

Figure 3 Graph showing the dependence of the density of water on the pressure (regression function (8) based on experimental results according to [25])

VI. The dynamic viscosity of water is dependent on the pressure, as seen in Fig. 4 [26]. In the estimated range up to $350 \mathrm{MPa}$, it will be approximated by a constant value of

$$
\eta=1,05 \times 10^{-3} \mathrm{~N} \cdot \mathrm{s} / \mathrm{m}^{2}
$$

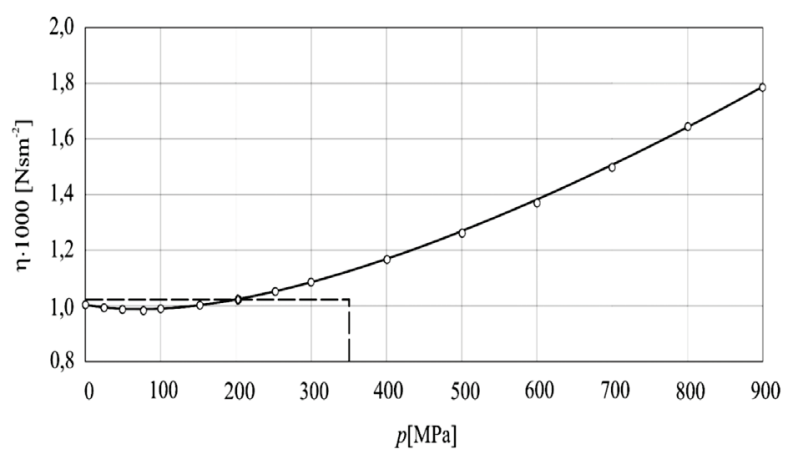

Figure 4 Graph showing the dependence of the dynamic viscosity of water on the pressure according to [26]

VII. For the maximum velocity of $v=200 \mathrm{~m} / \mathrm{s}$, the Reynolds number gets the value of 


$$
\operatorname{Re}=\frac{2 R|v| \rho}{\eta} \approx 188000
$$

In this mode, the flow is significantly turbulent.

VIII. The tube walls are considered to be hydrodynamically "smooth" and the loss coefficient of the turbulent flow $\lambda$ is defined as a proportionality constant in the Darcy-Weissbach equation for the amount of the pressure loss [27]

$$
\Delta p=\lambda \frac{\Delta x}{2 R}\left(\frac{1}{2} \rho v^{2}\right) \rightarrow \lambda \stackrel{\text { def }}{=} 2 R\left(\frac{\Delta p}{\Delta x}\right)\left(\frac{1}{2} \rho v^{2}\right)^{-1} .
$$

For anticipated flow velocities, the Blasius semiempirical relation holds for $\lambda$ [27]

$$
\lambda=\underset{\substack{\uparrow \\ 0,3164}}{\alpha} \cdot \operatorname{Re}^{-\frac{1}{4}} \text { to } \operatorname{Re} \approx 10^{5} .
$$

IX. For the loss coefficient $\lambda$ of the turbulent flow in the macroscopic profile, the impact of the wall effect on inter-phase tension between the liquid and the tube is not considered.

Assuming the above-listed conditions, the basis for the general formulation of a system of differential equations of a one-dimensional description of the hydraulic shock in a thin long shock tube is determined.

In the considered case, the non-stationary flow of the compressible liquid at the velocity $\boldsymbol{v}$ is subjected to the mass balance equation

$\left(\frac{\partial \rho}{\partial t}+\boldsymbol{v} \cdot \nabla \rho\right)+\rho \nabla \cdot \boldsymbol{v}=q$

Regarding the fact that the source term $q$ is equal to zero as a consequence of the mass conservation, the general balance equation for one-dimensional liquid flow gets the well-known form of the continuity equation in one dimension

$\left(\frac{\partial \rho}{\partial t}+v \frac{\partial \rho}{\partial x}\right)+\rho \frac{\partial v}{\partial x}=0$

For the liquid pressure $p$ and the velocity $v$, the Eq. (14) holds the general form of

$\frac{\partial \rho}{\partial t}\left(1+\frac{v}{a}\right)+\rho \frac{\partial v}{\partial x}=0$.

where the parameter $a$ represents the absolute velocity of the shock wave propagation in the tube

$\frac{\mathrm{d} x}{\mathrm{~d} t}=a$

Given the dependence of the density on the pressure (8), it is convenient to rewrite the continuity equation (15) in the final form of

$\left(\frac{1}{\rho} \frac{\mathrm{d} \rho}{\mathrm{d} p}\right) \frac{\partial p}{\partial t}+\left(1+\frac{v}{a}\right)^{-1} \frac{\partial v}{\partial x}=0$
The other governing equation for the hydraulic shock in the thin long tube is the momentum balance of the flowing liquid. The driving force of this motion is represented by the components of mechanical stresses $\boldsymbol{T}_{x}$, $\boldsymbol{T}_{r}, \boldsymbol{T}_{\varphi}$ of the stress tensor $\boldsymbol{T}_{i j}$

$$
\rho\left(\frac{\partial v}{\partial t}+\boldsymbol{v} \cdot \nabla \boldsymbol{v}\right)=\boldsymbol{e}_{j} \cdot \nabla \boldsymbol{T}_{j}
$$

With regard to the character of the flow (4) and (5) and the rotary symmetry of the problem, the general momentum balance (18) can be written in a specific form using the two non-zero components of the stress tensor

$$
\rho\left(\frac{\partial v_{x}}{\partial t}+v_{x} \frac{\partial v_{x}}{\partial x}\right)=\frac{\partial T_{x x}}{\partial x}+\frac{\partial T_{r x}}{\partial r}
$$

As a consequence of Pascal's law, the tensor component $T_{x x}$ corresponds to a negative liquid pressure $T_{x x}=-p$. The other non-zero component $T_{r x}$ corresponds to hydraulic friction losses of the viscous flow in a cylindrical tube. For a very thin liquid layer $\Delta x$ in a thin long tube, the Eq. (19) can be rewritten in an integral form as a balance of the total momentum of the liquid in the volume of $\pi R^{2} \Delta x$ :

$\Delta x \int_{0}^{R} \int_{0}^{2 \pi} \rho\left(\frac{\partial v_{x}}{\partial t}+v_{x} \frac{\partial v_{x}}{\partial x}\right) \mathrm{d} \varphi r \mathrm{~d} r=$

$-\Delta x \int_{0}^{R} \int_{0}^{2 \pi} \frac{\partial p}{\partial x} \mathrm{~d} \varphi r \mathrm{~d} r+\Delta x \int_{0}^{R} \int_{0}^{2 \pi} \frac{\partial T_{r x}}{\partial r} \mathrm{~d} \varphi r \mathrm{~d} r$.

Regarding the conditions (4), (5) and (7), the Eq. (20) can be significantly simplified to the form of

$\rho\left(\frac{\partial v_{x}}{\partial t}+v_{x} \frac{\partial v_{x}}{\partial x}\right) R^{2}=-\frac{\partial p}{\partial x} R^{2}+2 \int_{0}^{R} \frac{\partial T_{r x}}{\partial r} r \mathrm{~d} r$

In accordance with the boundary layer theory $\delta_{m} \ll R$, the radial dependence of the friction component $T_{r x}$ can be approximated by a piecewise linear function

$$
\begin{aligned}
& T_{r x}=-\frac{v_{x}}{\left|v_{x}\right|} \frac{T_{0}}{\delta_{m}} r-R-\delta_{m} \text { for } R-\delta_{m} \leq r \leq R \\
& T_{r x}=0 \quad \text { for } 0 \leq r \leq R-\delta_{m}
\end{aligned}
$$

where $T_{0}$ represents the friction stress at the wall of the tube with radius $R$. Having substituted the function (22) the Eq. (21) obtains the form of

$$
\begin{aligned}
\rho\left(\frac{\partial v_{x}}{\partial t}+v_{x} \frac{\partial v_{x}}{\partial x}\right) & R^{2}= \\
& =-\frac{\partial p}{\partial x} R^{2}-\frac{2 v_{x}}{\left|v_{x}\right|} \frac{T_{0}}{\delta_{m}} \int_{R-\delta_{m}}^{R} r \mathrm{~d} r
\end{aligned}
$$

and after the integration and neglecting of the minority term of second order $\delta_{m}^{2}$, the following holds

$\rho\left(\frac{\partial v_{x}}{\partial t}+v_{x} \frac{\partial v_{x}}{\partial x}\right)+\frac{\partial p}{\partial x}+\frac{2 T_{0} v_{x}}{R\left|v_{x}\right|}=0$ 
If $v_{x}$ denotes the mean cross-sectional velocity $v$ and the left side is expressed in the analogical form as in the Eq. (15), the equation of motion obtains the onedimensinal form of

$\rho \frac{\partial v}{\partial t}\left(1+\frac{v}{a}\right)+\frac{\partial p}{\partial x}+\frac{2 T_{0} v}{R|v|}=0$

The so far undetermined value of the friction stress $T_{0}$ at the wall of the tube can be expressed from the equation of motion (25) for a steady flow $\partial v / \partial t=0$

$T_{0}=-\frac{R}{2} \frac{\partial p}{\partial x} \frac{|v|}{v}=\frac{R}{2}\left|\frac{\partial p}{\partial x}\right|$

Having applied the relations (10), (11) and (12), the Eq. (26) obtains the specific form of

$T_{0}=\frac{\alpha}{8} \rho v^{2}\left(\frac{\eta}{2 R|v| \rho}\right)^{\frac{1}{4}}$

that will be used for the final formulation of the equation of motion (25)

$$
\left(1+\frac{v}{a}\right) \frac{\partial v}{\partial t}+A|v|^{\frac{3}{4}} v+\frac{1}{\rho} \frac{\partial p}{\partial x}=0
$$

For the above-mentioned formulation, the coefficient of the loss term $A$ assumes the value of

$A=\left(\frac{\alpha^{4} \eta}{512 \rho R^{5}}\right)^{\frac{1}{4}} \doteq 28,5$

It is useful to describe the basic wave process, given by the governing Eqs. (17) and (28), in the case of a nonviscous flow in first approximation to the continuity equation

$$
\frac{\partial v}{\partial x}+\left[\left(1+\frac{v}{a}\right) \frac{1}{\rho} \frac{\partial \rho}{\partial p}\right] \frac{\partial p}{\partial t}=0
$$

and the equation of motion without the loss term $(A \rightarrow 0)$

$$
\frac{\partial p}{\partial x}+\left[\left(1+\frac{v}{a}\right) \rho\right] \frac{\partial v}{\partial t}=0
$$

The specification of the parameter of the velocity of shock wave propagation $a$ comes from the experience in the dynamics of acoustic waves, where the amplitudes of the density $\Delta r$ and the velocity $\Delta v$ represent a mere fragment of their mean values $\rho\left(p_{0}\right)$ and $v\left(p_{0}\right)$ at the equilibrium pressure $p_{0}$. In this special case, the pressure dependence (8) can be linearized in a small neighbourhood $\rho\left(p_{0}\right)$ as

$\rho(p)=\rho\left(p_{0}\right)+\Delta p=\rho\left(p_{0}\right)+\frac{\partial \rho\left(p_{0}\right)}{\partial p}\left(p-p_{0}\right)$

and in case of a small deviation of pressure, it can be neglected. With respect to the Bernoulli equation of conservation of energy density, where the friction and deformation dissipations are neglected

$p_{0}+\frac{1}{2} \rho\left(p_{0}\right) v_{0}^{2} \approx p+\frac{1}{2} \rho\left(p_{0}\right) v^{2}$,

it is possible to apply the similar approximation also for the velocity. Having used the approximate relation for the difference between squares of velocities $\left(v_{0}^{2}-v^{2}\right) / 2 \approx$ $v_{0}\left(v_{0}-v\right)$, the following equation is obtained from the relation (33)

$$
v(p)=v_{0}+\Delta v=v_{0}+\left[\frac{-1}{\rho\left(p_{0}\right) v_{0}}\right]\left(p_{0}-p\right)
$$

If the liquid pressure at the point $\left(x_{0}, t_{0}\right)$ achieves the value $p_{0}$ and the liquid velocity the value $v_{0}$, then the terms in square brackets of governing Eqs. (30) and (31) can be considered to be constant for small changes in pressure $p$ and in velocity $v$ and it can be written

$\frac{\partial v}{\partial x}+\left[\frac{\left(a+v_{0}\right)}{\rho\left(p_{0}\right) a} \frac{\partial \rho\left(p_{0}\right)}{\partial p}\right] \frac{\partial p}{\partial t}=0$

$\frac{\partial p}{\partial x}+\left[\frac{\left(a+v_{0}\right) \rho\left(p_{0}\right)}{a}\right] \frac{\partial v}{\partial t}=0$.

Deriving the Eq. (35) along $t$ and the equation (36) along $x$, and after subsequent elimination of $\partial_{x} \partial_{t} v$, the wave equation for the pressure $p$ is as follows

$\frac{\partial^{2} p}{\partial x^{2}}-\left[\frac{\left(a+v_{0}\right)^{2}}{a^{2}} \frac{\partial \rho\left(p_{0}\right)}{\partial p}\right] \frac{\partial^{2} p}{\partial t^{2}}=0$.

The term in square brackets in the wave equation has the meaning of the reciprocal square value of the wave velocity $a$

$$
\frac{\left(a+v_{0}\right)^{2}}{a^{2}} \frac{\partial \rho\left(p_{0}\right)}{\partial p}=\frac{1}{a^{2}}
$$

Assuming the validity of the Eq. (38), the lower index " 0 " can be omitted for all values $p_{0}$ and $v_{0}$, and the following holds for the value $a$

$a=a_{0}(p)-v, \quad a_{0}(p)= \pm \sqrt{\frac{\partial p}{\partial \rho(p)}}$

While an alternative sign defines the specific orientation of wave propagation along the $x$ axis, the parameter $a_{0}(p)$ determines the dependence of the absolute velocity of the wave on the pressure (see Fig. 5).

The above-mentioned relation makes obvious that the maximum velocity of the pressure wave peak deforms the shape of the wave and causes formation of a very steep front with an extreme pressure gradient. 


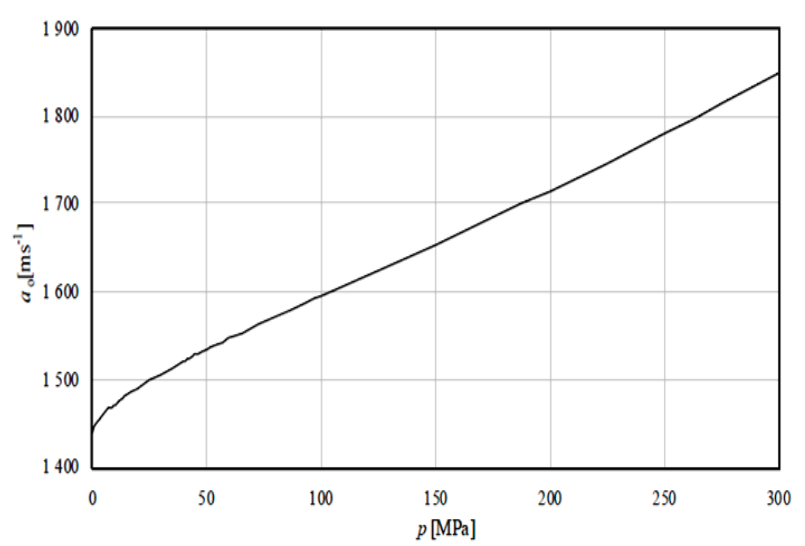

Figure 5 The graph of dependence of the velocity of a pressure wave in water on the ambient pressure according to the Eq. (39)

The mechanism of a hydraulic shock was described in quantitative terms for the first time in the work [24] by Zhukovsky using a relatively simple equation for the maximum amplitude $p$ (or $\Delta p$ ) of the pressure wave during a total shock. The classical Zhukovsky formula, which has been practically proven for many times, is a good approximation used for not too high pressure amplitudes, where the pressure dependence of the pressure wave velocity is not significant. In a more general case, where the condition of validity of the abovementioned approximation $a=$ const. is not fulfilled, the problem of the amplitude of the total shock is solved in the first approximation from the integration of the equation of motion (31)

$\frac{\partial v}{\partial t}=-\frac{a}{(a+v) \rho} \frac{\partial p}{\partial x}$

Assuming that the velocity of the pressure wave $a$ is the function of the pressure the equation of motion (40) can be formulated, applying (16) and (39), in the specific integrable form of

$\frac{\partial v}{\partial t}=-\frac{1}{\rho} \sqrt{\frac{\partial \rho}{\partial p}} \frac{\partial p}{\partial t}$.

The subsequent integration provides the resulting formula for the axial acoustic velocity $v$ of the total hydraulic shock in the general form of

$v=\int_{0}^{p} \frac{1}{\rho} \sqrt{\frac{\partial \rho}{\partial p}} d p$

and in the special case for water

$v=\frac{1}{3 \sqrt{\rho_{0}}} \int_{0}^{p} \mathrm{e}^{-\gamma_{0} p+\beta p^{\frac{5}{3}}}\left(3 \gamma_{0}-5 \beta p^{\frac{2}{3}}\right) \mathrm{d} p$.

In Fig. 6, shapes of the integral function (43) for water (solid curve) and the classical Zhukovskij prediction calculated according to the Eq. (2) (dashed linear relation) are compared.

As can be seen in Fig. 6, at the initial maximum velocity of the water stream of $200 \mathrm{~m} / \mathrm{s}$, the real pressure amplitude and the Zhukovsky linear prediction differ by approx. $75 \mathrm{MPa}$. However, to obtain a continuous velocity field of $200 \mathrm{~m} / \mathrm{s}$ using a standard setup for the generation of a hydraulic shock with high-speed flow through a long tube is technically infeasible. Therefore, it is necessary to generate it in a pulse mode using a pulse multiplication technique, as described below.

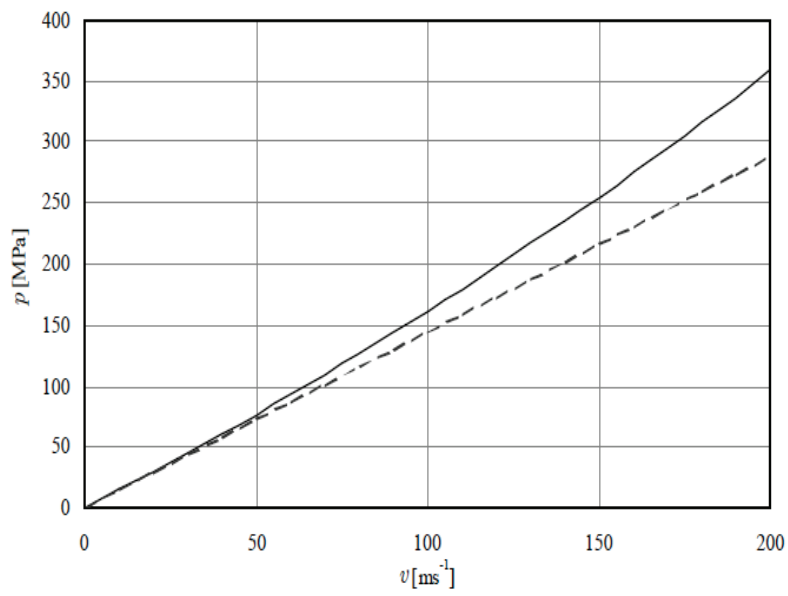

Figure 6 The relation between the pressure amplitude $p$ of the hydraulic shock and the velocity $v$ of the liquid calculated according to the Eq. (43) -solid line, and according to the classical Zhukovskij equation dashed line

\section{Application of hydraulic shock for pulsed liquid jet generation}

The practical objective of this chapter is to present a new "pulse modulation technique" [11] that can be classified to the category of periodically operationing "pulse guns" according to its technical design. The principle of the pulse multiplication is based on impacts of the front of a periodically interrupted primary jet at the outflow velocity of $v \approx 200 \mathrm{~m} / \mathrm{s}$ into an inlet of a thin high-pressure shock tube. The tube ends with a nozzle for generation of the water jet or with a high-pressure sensor for the purpose of studies. At the moment of impact, a part of impacting liquid is stopped extremely quickly, which leads to the transformation of the kinetic energy into the high-pressure hydraulic shock inside the shock tube (the so-called water hammer effect). According to the above-mentioned Eq. (43), theoretical pressure maximums at the above-mentioned primary velocity can be expected in the order of hundreds of $\mathrm{MPa}$ with a very steep front of the shock wave. At the nozzle level, the shock wave causes the injection of a limited dose of the liquid with a high output velocity. In contrast to the principles of modulation that require high frequencies, the frequency of individual shocks is not limited by the condition of fast kinetics of the jet decomposition in the short distance behind the nozzle, but it is related to wave phenomena inside the shock tube and the requirement of the maximum output power. The working name "pulse multiplication" can be considered to be justified, as the input pressure in the pulse mode is theoretically amplified up to 15 times.

Based on the above-described physical analysis of the problem of the generation of hydraulic shocks in the water, we have been awarded the patent [11] for "The method of generation of high-pressure pulses in the liquid 
using the technique of pulse multiplication and the device for implementation of this technique", where the "method of pulse multiplication" and design principles for operations of the technical generator of high-pressure pulses, the so-called "pulse multiplier", were formulated.

\subsection{Classical hydraulic shock and problem of hydraulic resistance during technical application}

Classical hydraulic shock described by the continuity Eq. (17) and the equation of motion (28) is traditionally considered to be a stream of liquid with the maximum cross-sectional velocity of approx. $2 \mathrm{~m} / \mathrm{s}$ limited in radial direction by the walls of the tube. The primary shock wave spreads in the opposite direction to the direction of the original flow after a very fast closing of the outflow end of the tube. According to the Eq. (43), the crosssectional velocity of at least approx. $170 \mathrm{~m} / \mathrm{s}$ would be necessary for the generation of the total high-pressure shock in the order of $300 \mathrm{MPa}$. So far, this is technically feasible with extremely high energy losses and using the tubes of very short lengths only. For these reasons, the hydraulic shock with the above-described standard setting-up is applied to well-known water rams exclusively at low flow velocities.

\subsection{Impact of front of fast liquid jet into the inlet of shock tube}

In the above-mentioned pulse multiplier, the hydraulic shock is generated in different ways. A medium-pressure water pump with a sufficient flow and a limit pressure of $25 \mathrm{MPa}$ generates a primary water jet with the velocity of about $200 \mathrm{~m} / \mathrm{s}$ in the output nozzle. Due to a mechanical interrupter, the water jet front impacts periodically into an open inlet of the shock tube of the similar inner diameter that ends on the opposite side by a very thin high-speed nozzle of a diameter of $d_{t r}$ $=0,13 \mathrm{~mm}$. At every impact of the front of the primary jet, the shock wave is initiated in the inlet of the shock tube in form of a very short column of water with the starting velocity of $200 \mathrm{~m} / \mathrm{s}$. Thus the initial boundary value condition for solving the system of governing Eqs. (17) and (28) is practically fulfilled. It is obvious that in the aforementioned setting, the resistive losses of the primary jet are many times lower than in case of the flow in the tube during the classical shock.

\subsection{Problem of hydraulic losses in shock tube during the outflow of the pressure liquid supplying nozzle of high- energy water jet}

When the pressure wave reaches the closed end of the shock tube, it is reflected in the opposite direction and returns back towards the inlet. If the whole cross-section of the inlet is filled with the impacting primary jet, then the total hydraulic shock with the maximum pressure amplitude of $p_{\max } \approx 350 \mathrm{MPa}$ is generated, as seen in Fig. 6. When the shock tube ends with a thin highpressure nozzle with the hydraulic contraction factor of $\kappa \approx 0,5$, then the impact of the shock wave causes an injection of the pulsed water jet with the velocity maximum at the front of the jet of $\left(v_{\text {noz }}\right)_{\max }=\kappa \sqrt{\frac{2\left(p_{\max }=350 \mathrm{MPa}\right)}{\left(\rho\left(p_{\max }\right)=1110 \mathrm{~kg} / \mathrm{m}^{3}\right)}}$
$\left(v_{\text {noz }}\right)_{\max } \approx 400 \mathrm{~m} / \mathrm{s}$

If the shock tube at its end should "supply pressure" for the periodically pulsed water jet, then the continuity of flow between the shock tube of a diameter of $1 \mathrm{~mm}$ and the secondary water nozzle of a diameter of $d_{n o z}=$ $0,13 \mathrm{~mm}$ (assuming hypothetical conservation of density of the liquid at the front of the shock wave) implies the maximum cross-sectional velocity in the shock tube of

$v_{\max }^{k}=\left[\frac{\left(d_{t r}=0,13 \mathrm{~mm}\right)}{(2 R=1 \mathrm{~mm})}\right]^{2} \cdot\left(\left(v_{\text {noz }}\right)_{\max } \approx 400 \mathrm{~m} / \mathrm{s}\right)$

$v_{\max }^{k} \approx 6,76 \mathrm{~m} / \mathrm{s}$,

that corresponds to the average convective flow of a liquid column inside the tube. The intrinsic hydrodynamic description of individual shock waves is given by the coordinate system moving along the $x$-axis at the average velocity of the convective flow $v_{k_{\max }}$. It is obvious that this translation transformation does not significantly affect the value of the acoustic velocity maximum of the liquid in the shock wave. Due to its relatively high values, it is not possible to neglect the friction loss term of $A=28,5$ in the equation of motion (28), and the acoustic velocity $V$ of the liquid in the shock wave is described by a nonhomogeneous wave equation with strongly non-linear term of the loss. It strongly dampens the periodical motion of the shock wave inside the shock tube and only the first maximum will be technically applicable.

\section{Technical design of pulse multiplier}

From the technical point of view, the pulse multiplier has the form of a thin long tube with a circular crosssection of the inner diameter of $2 R=1 \mathrm{~mm}$ and the length of $L \gg 2 R$ (see Fig. 7). The total volume of the tube is filled with the liquid. The beginning of the tube serves as an inlet point for the impact of the front of the primary water jet at the velocity of $v_{0} \approx 200 \mathrm{~m} / \mathrm{s}$. On the opposite side, the tube ends with a high-speed sapphire nozzle of the diameter of $d_{n o z}=0,13 \mathrm{~mm}$ or with a highpressure sensor, if measurements are made.

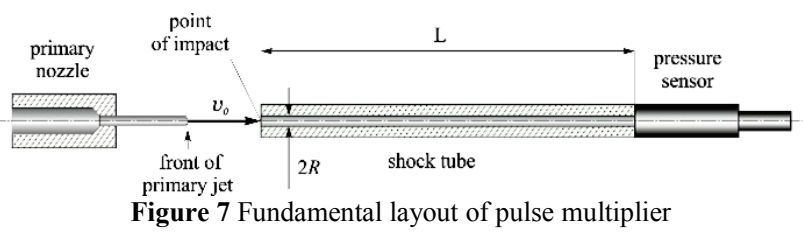

The hydraulic shock is generated in the shock tube just after the impact of the front of the primary jet into the inlet of the tube. For the application of the pulse multiplier in practice, fast periodical repetition of the impact is essential. The first experimental pulse multiplier was designed and built in accordance with these requirements, see Fig. 8.

A cylindrical body of the primary nozzle is tightly inserted into the fixed pump output $(0 \div 25 \mathrm{MPa})$. The outcoming primary jet is interrupted by a thin barrier that 
moves traversally between two parallel channels in the housing interrupter due to an intermittent tension of two solenoid coils. The periodically interrupted front of the primary jet impacts into the shock tube inlet and the kinetic energy cumulated by its deceleration initiates the hydraulic shock inside the shock tube. The rest of the liquid flows around the inlet and is drained off into a wide drain tube by four outlet ports. The beginning of this tube is slightly indicated in Fig. 8.

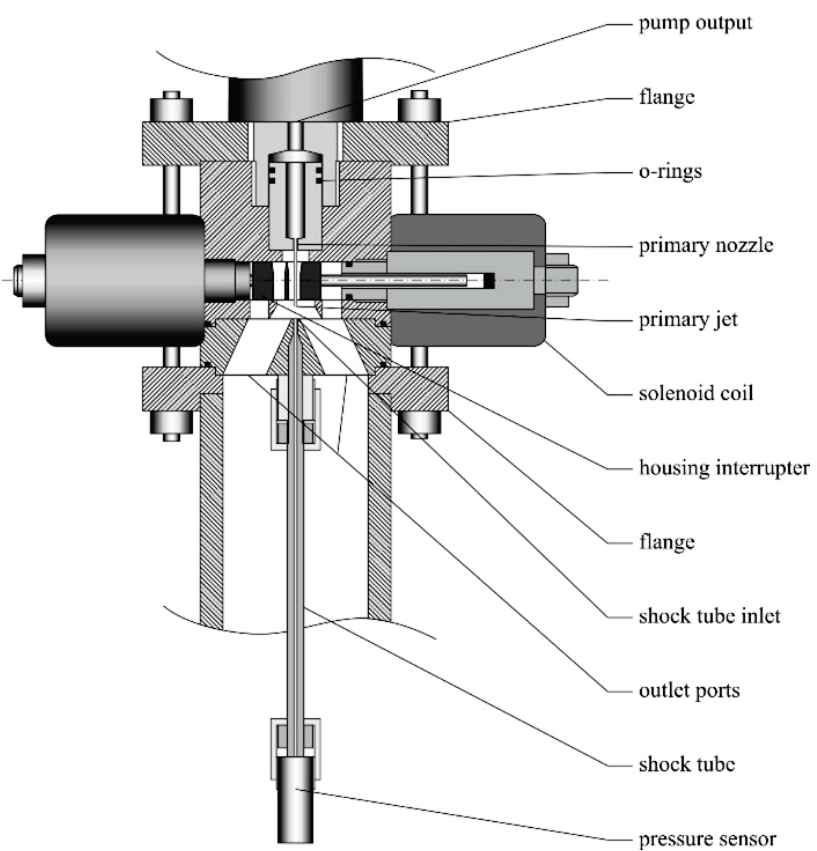

Figure 8 Technical solution of the first version of experimental pulse multiplier

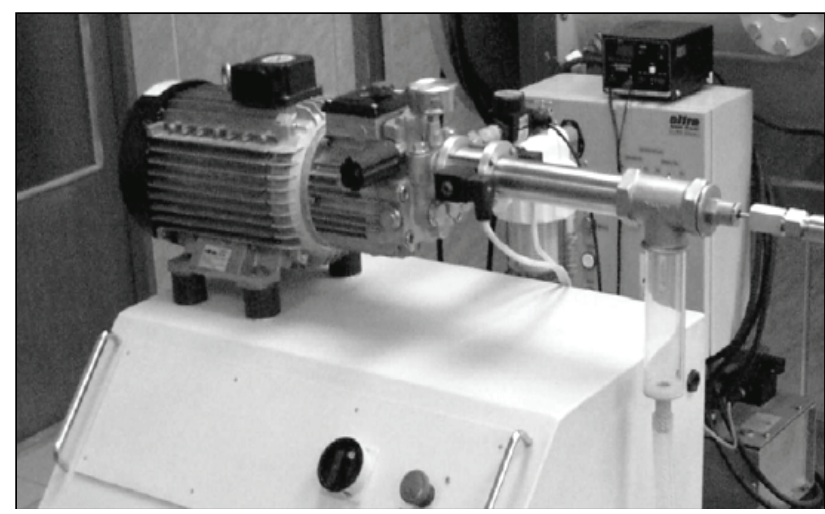

Figure 9 First experimental version of pulse multiplier with small Krenzle 3270 pump ( $25 \mathrm{MPa} / 13,51 / \mathrm{min})$ and KISTLER 4067A 5000

A2 piezoresistive sensor at the end of the shock tube (right side)

The whole system of the pulse multiplier, as shown in Fig. 8 and Fig. 9, has been tested at the input water pressure of $20 \mathrm{MPa}$ and the occurrence of the pulse multiplication effect has been proven. The effect was registered despite a very short length of the shock tube $(400 \mathrm{~mm})$ that practically does not enable the generation of the total hydraulic shock.

At present, an experimental study of the pulse multiplier system is based on measured responses of an isolated impact of the primary jet front into the shock tube inlet. Due to a stochastic nature of the coincidence of interruptions with maximum pressures generated by the three-piston pump, the resulting pulses were of significantly varied character (this defect will be eliminated by adding a hydraulic accumulator in front of the system). One of the positive cases of recording the pulse maximum of $66 \mathrm{MPa}$ is shown in Fig. 10.

Unfortunately, the graph showing the time course of the measurement of the highest recorded value of $80 \mathrm{MPa}$ was not preserved. In case of the short length of the shock tube $(400 \mathrm{~mm})$ when achieving the total hydraulic shock is very problematic, this result indicates a significant application potential of the system. At relatively slow mechanical interruptions, pressure maximum values generated in the short tube are much lower than the theoretical value of the total shock. The period of shock waves in the shock tube of the length given above, as clear from the graph, is approximately $2,5 \mathrm{~ms}$. This period should be much longer in longer tubes. This should allow to synchronise periodic impacts of the primary jet with the maximum values of reflected impacts and thus to achieve an additional feedback amplification.

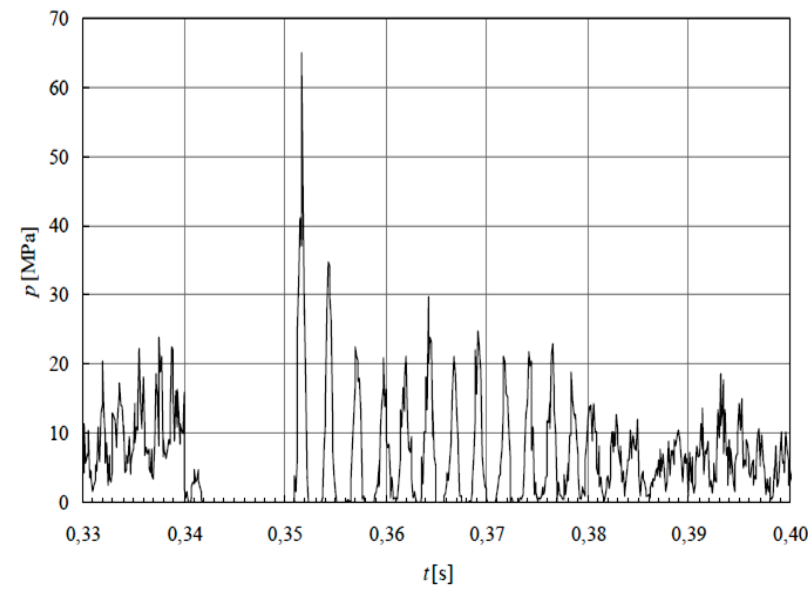

Figure 10 Time behaviour of shock with maximum of $66 \mathrm{MPa}$ measured by KISTLER 4067A 5000 A2 piezoresistive sensor

\section{Conclusions}

Regarding requirements for further increase of the disintegration effect of the high-energy water jet without the addition of abrasive particles, an experiment to set up a pulsed system of high-pressure generation was performed. The system should allow the use of high energy, cumulated as a result of the water hammer effect of very fast drops of the pulsed jet, for improvement of the disintegration effect of the jet. The generalisation of the classical hydraulic shock theory for high pressures is presented in the paper. The generalisation is subsequently applied for the formulation of the principle of pulse multiplication. This is a technical solution allowing the generation of extreme pressure pulses of the hydraulic shock at velocities of the primary flow of approx. $200 \mathrm{~m} / \mathrm{s}$. The mentioned flow is generated in a short inlet section of the so-called shock tube by periodic impacts of the primary low-pressure jet $(20 \mathrm{MPa})$ with the identical output velocity. In this way, a shock wave in the order of hundreds of $\mathrm{MPa}$ arises in the shock tube. This wave is several times higher than the stagnation pressure of the primary low-pressure jet. The source of high-pressure pulses is a technical device "pulse multiplier" that enables supply of nozzle of a pulsed liquid jet with $100 \%$ depth of outlet velocity modulation. Thus the disintegration 
effect of the device can be significantly improved without addition of abrasive materials.

\section{Acknowledgments}

Presented work was supported by the project IA2 "MATERIALS" - OP VaVpI (7.3-PO3) and Regional Materials Science and Technology Centre (CZ.1.05/2.1.00/01.0040) in Ostrava, the project of the Grant Agency of the Czech Republic No. 15-23219S and the project for support of long-term strategic development of research organization RVO: 68145535.This article was also written within the scope of a project of the Institute of Clean Technologies for Mining and Utilization of Raw Materials for Energy Use - Sustainability Program, reg. no. LO1406, which is supported by the Research and Development for Innovations Operational Programme financed by the Structural Funds of the European Union and the State Budget of the Czech Republic. Authors are thankful for the support.

\section{References}

[1] Summers, D. A. Waterjetting Technology. E \& FN SPON, an Imprint of Chapman \& Hall, London, 1995. DOI: 10.4324/9780203475317

[2] Howells, W. G. Super water jetting applications from 1974 to 1999. http://www.berkeleychemical.com/download/ SUPER-WATER.pdf (01.10.2013).

[3] Rice, R. M. Process Report on the Automated Hydro Removal of TPS prepared for NASA, Contract NAS836300,1986

[4] Hoppe, D. T.; Babai, M. K. High pressure water jet cutting and stripping. NASA Center: Marshall Space Flight Center 1991. http://ntrs.nasa.gov/archive/nasa/casi. ntrs.nasa.gov/ 19910013734 199101 3734.pdf (01.10.2013)

[5] Brennen, $\mathrm{CH}$. E. Fundamentals of Multiphase Flow. Cambridge University Press, 2005. DOI: 10.1017/CBO9780511807169

[6] Young, R. F. Cavitation. Imperial College Press, 1999.

[7] Joseph, D. D. Cavitation in a flowing liquid. // Phys. Rev. E. 51, 3(1995), pp. 1649-1650. DOI: 10.1103/PhysRevE.51.R1649

[8] Joseph, D. D. Cavitation and the state of stress in a flowing liquid. // Journal of Fluid Mechanics. 366, (1998), pp. 367378. DOI: $10.1017 /$ S0022112098001530

[9] Vijay, M. M. Ultrasonically generated cavitating or interrupted jet. (Gloucester, CA) 1992. US Patent 5154347.

[10] Foldyna, J.; Sitek, L.; Svehla, B.; Svehla, S. Utilization of ultrasound to enhance high-speed water jet effects. // Ultrasonics Sonochemistry. 11, 3-4(2004), pp. 131-137. DOI: 10.1016/j.ultsonch.2004.01.008

[11] Dvorsky, R. Method of generation of high-pressure pulses in a liquid using the technique of pulse intensification and the equipment for the application of this technique. In Czech. (Způsob vytváření vysokotlakých pulzů v kapalině metodou pulzní multiplikace a zařízení $\mathrm{k}$ provádění tohoto způsobu.) 2007, CZ Patent 298 759/2007.

[12] Foldyna, J.; Sitek, L.; Habán, V. Acoustic wave propagation in high-pressure system. // Ultrasonics. 44, (2006), pp. e1457-e1460. DOI: 10.1016/j.ultras.2006.05.144

[13] Ř́ha, Z.; Foldyna, J. Ultrasonic pulsations of pressure in a water jet cutting tool. // Technical Gazette. 19, 3(2012), pp. 487-491.

[14] Heymann, F. J. High-Speed Impact betveem a Liquid Drop and a Solid Surface. // Journal of Applied Physics. 40, 13(1969), pp. 5113-5122. DOI: 10.1063/1.1657361
[15] Huang Y. C.; Hammitt F. T.; Yang W. J. Mathematical Modelling of Normal Impact between a Finite Cylindrical Liquid Jet and Non-Slip, Flat Rigid Surface. // Proceedings of the $1^{\text {st }}$ International Symposium on Jet Cutting Technology / Cranfield, 1972, pp. A4-57 - A4-68.

[16] Smith, D. G.; Kinslow, R. Pressure Due to High-velocity Impact of a Water Jet. // Experimental Mechanics. 16, 1(1976), pp. 21-25. DOI: 10.1007/BF02328917

[17] Kollé, J. J. Development and Applications of Hydraulic Pulse Generator. // Proceedings of the $7^{\text {th }}$ American Water Jet Conference / St. Louis, 1993, pp. 459-471.

[18] Lichtarowicz, A.; Nwachukwu, G. O. Erosion by an Interrupted Jet. // Proceedings of $4^{\text {th }}$ Int. Symposium of Jet Cutting Technology / Cranfield, 1978, pp. B2-13 - B2-18.

[19] Rayleigh, W. S. On the instability of jets. // Proceedings of the London Mathematical Society / London, 1878, pp. 4-15. DOI: 10.1112/plms/s1-10.1.4

[20] Pimbley, W. T. Drop Formation from a Liquid Jet: A Linear One-Dimensional Analysis Considered as a Boundary Value Problem. // IBM J. Research Development. 20, 2(1976), pp. 148-156. DOI: 10.1147/rd.202.0148

[21] Sami, C.; Anderson, C. Helmoltz Oscillator for the SelfModulation of a Jet. // Proceedings of the $7^{\text {th }}$ Int. Symposium of Jet Cutting Technology / Cranfield, 1984, pp. B4-91 - B4-98.

[22] Shen, Z. H.; Wang, Z. M. Theoretical analysis of a jetdriven Helmholtz resonator and effect of its configuration on the water jet cutting property. // Proceedings of the $9^{\text {th }}$ Int. Symposium of Jet Cutting Technology/ Cranfield, 1988, pp. D4-189 - D4-201.

[23] Foldyna, J.; Švehla, B. Method of generation of pressure pulsations and apparatus for implementation of this method. 2008, US Patent 2008/0135638 A1.

[24] Zhukovsky, N. J. O gidravličeskom udare v vodoprovodnych trubach, 1899. Polnoje sobranije sočinenij, Moscow-Leningrad, 1935, sv.VII (in Russian).

[25] Valouch, M. Pětimístné logaritmické tabulky a tabulky konstant, SNTL, Praha, 1967 (in Czech).

[26] Bett, K. E.; Cappi, J. B. Letter to editor - Effect of Pressure on the Viscosity of Water. // Nature. 207, 4997(1965), pp. 620-621. DOI: 10.1038/207620a0

[27] Smetana, J. Hydraulika, ČSAV, Praha, 1957.

\section{Authors' addresses}

Richard Dvorsky, Assoc. prof. RNDr. PhD

VŠB - Technical University of Ostrava, Department of Physics, Regional Materials Science and Technology Centre,

17. listopadu 15/2172, 70833 Ostrava-Poruba, Czech Republic Tel.: +420 731186 696, richard.dvorsky@vsb.cz

\section{Libor Sitek, Ing. PhD}

Institute of Geonics of the AS CR, v.v.i., Department of material disintegration,

Studentská 1768, 70800 Ostrava-Poruba, Czech Republic Tel.:+420 596976 323, libor.sitek@ugn.cas.cz

Tomáš Sochor, RNDr. CSc.

University of Ostrava,

Department of Informatics and Computers, 30. dubna 22, 70103 Ostrava, Czech Republic Tel.: +420 553462 129, tomas.sochor@osu.cz 


\section{MODELSWARD 2017}

$5^{\text {th }}$ International Conference on Model-Driven Engineering and Software Development

$$
\text { Porto, Portugal 19- } 21 \text { February, } 2017
$$

Frameworks for Model-Driven Development

Model Transformations and Generative Approaches Business Process Modeling

Domain-specific Modeling Executable UML

Model Execution and Simulation

Model-Driven Architecture

Model-based Testing and Validation Systems Engineering

Reasoning about Models

$$
\text { Generative Programming Software Process Modeling, Enactment and Execution }
$$

Model Transformation Constraint Modeling and Languages Workflow Management Systems

Agile Model-Driven Development Syntax and Semantics of Modeling Languages

General-purpose Modeling Languages and Standards Service Oriented Architectures

Meta-modeling: Foundations and Tools

\section{CONFERENCE CHAIR}

Bran Selic, Malina Software Corp., Canada

\section{PROGRAM CO-CHAIRS}

Luis Ferreira Pires, University of Twente, Netherlands

Slimane Hammoudi, ESEO, MODESTE, France

\section{LOCAL CHAIR}

Ana Cristina Ramada Paiva, Faculty of Engineering of University of Porto, Portugal

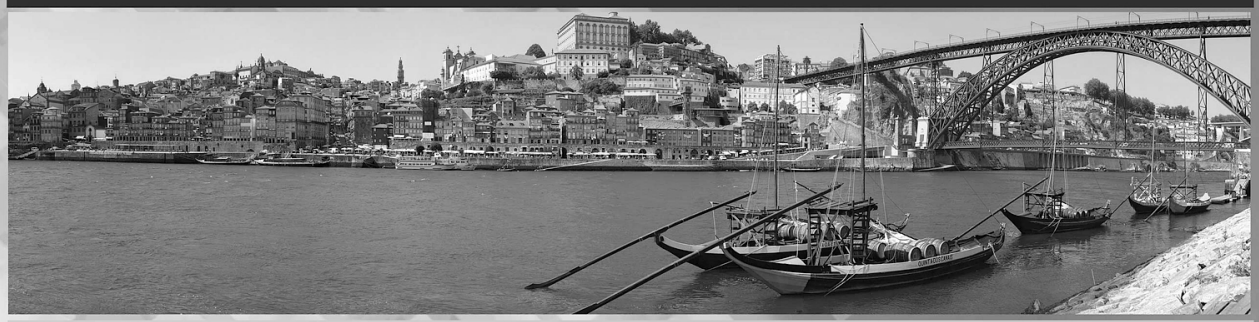

REGULAR PAPER SUBMISSION: OCTOBER 7, 2016

MORE INFORMATION AT: WWW.MODELSWARD.ORG

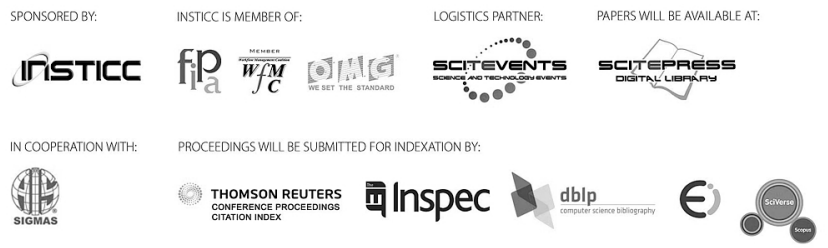

